\title{
THE ESTIMATION OF $\gamma$-GLOBULIN
}

\author{
BY \\ GEORGE DISCOMBE, RAYMOND F. JONES, AND D. P. WINSTANLEY \\ From the Central Middlesex Hospital, London \\ With Statistical Assistance From \\ C. DALY \\ From the Social Medicine Research Unit, Medical Research Council, London
}

(RECEIVED FOR PUBLICATION MARCH 15, 1954)

Filter-paper electrophoresis appears to be a technique suitable for the routine laboratory; the apparatus is simple and cheap, and although the electric current must be applied for 12 to 24 hours it is easy to arrange for this to occupy the night. During the last two years we have studied this technique, and conclude that the advantages are to some degree illusory. By this we do not mean that the information gained is useless, but that much of the useful information can be obtained by less laborious methods.

Martin and Franglen on page 87 of this issue write a critical review of the subject, and no detailed bibliography will be given. We must emphasize that in classical electrophoresis protein concentrations are estimated from differences in refractive index: this is a non-specific property, and is affected by serum-lipoids which migrate chiefly with the $\alpha_{2}$ - and $\beta$-globulins, thus rendering estimates of the protein components fallacious. On filter-paper, the choice of a suitable dye should enable one to estimate true protein values (Loeffler and Wunderly, 1953).

It is easy, with any one of a series of dyes, to produce an electrophoresis strip which permits visual estimation of the proportions of the different protein components. It is much more difficult to obtain an objective measure of these protein components : the most reliable, but most laborious methods, are those in which the dye is eluted from segments of strip (Loeffler and Wunderly, 1953). Scanning methods, in which the light transmitted by the strip is measured, are less laborious but less reliable, for the very complex optical problem, in which absorption by dyestuff is superimposed on absorption and scatter by cellulose fibre immersed in air $(\mu=1.0)$ or organic solvent $(\mu=1.4-1.7)$, has been studied only by Crook, Harris, Hassan, and Warren (1954).

We concluded that there was a need for the examination of a very simple technique, and this paper describes such a technique and some of the results obtained. The most important result is that we find a very close correlation $(=+0.9143$ based on 45 pairs of observations) between Kunkel's (1947) zinc sulphate turbidity test and our electrophoretic estimates of $\gamma$-globulin. This agrees with Kunkel's own results when using classical electrophoresis. Since it is well known that changes in $\gamma$-globulin occur in a great variety of diseases, including liver disease, rheumatoid arthritis and other collagen diseases, some reticuloses, kidney disease, and myelomatosis, we consider that Kunkel's test is the most important of the non-specific " turbidity" tests, since it gives so close an estimate of $\gamma$-globulin, and we think that for most routine purposes it should do away with any need for electrophoresis.

\section{Procedure}

Electrophoretic Separation.-A strip of Whatman No. 1 filter paper, $5 \mathrm{~cm}$. wide and $50 \mathrm{~cm}$. long, is fixed in a perspex holder so arranged that the electric current is led in on two lines $45 \mathrm{~cm}$. apart. A middle section, $28 \mathrm{~cm}$. long, is held horizontal and approximately $7 \mathrm{~cm}$. above the buffer solution in the electrode vessels. The buffer solution is sodium barbitone, $0.05 \mathrm{M}$, and barbitone, $0.01 \mathrm{M}$, saturated with chloroform. When the paper has been placed in electric contact, soaked with buffer and allowed to come into gravitational equilibrium, serum $(0.02 \mathrm{ml}$.) is applied in a streak about $45 \mathrm{~mm}$. long, approximately $10 \mathrm{~cm}$. from the negative end of the horizontal paper. The electric current is switched on (175 to 200 volts, 0.6 $\mathrm{mA}$ per paper) and left on for 20 to 22 hours.

Fixing and Staining.-The wet strip is cut off the holder and dropped into the combined fixing and staining solution, containing $0.133 \%$ light green S.F. (Lissamine Green S.F. 130 from I.C.I. Dyestuffs, Ltd., colour index 670) in $3 \%$ salicylsulphonic acid. It is left for between 30 and 120 minutes, and then washed for five minutes or more in each of three changes of 
$\frac{1}{2}-2 \%$ acetic acid in tap water, finally being hung up to dry in front of a gas or electric radiant.

Light green S.F. was first suggested by Dr. W. G. Dangerfield ; I.C.I. Dyestuffs, Ltd., suggested that Lissamine violet 2R 124 (Victoria violet, colour index, 53) disulphine blue V.N. 150 (colour index, 712), Coomassie brilliant blue F.F.1 200 or crystal violet (colour index, 681) might also have the qualities required; that is, to dye proteins substantively from an aqueous acid bath, but to be immediately discharged from cellulose by dilute aqueous acid. It was found that the light green was the most effective of these dyes.

Recording.-In many cases, a simple inspection will provide the desired information, for example, in myelomatosis with increased $\gamma$-globulins, in nephrotic nephritis with an increase in $\alpha_{2}$-globulin; gross increases in $\gamma$-globulins or decreases in albumin can also be recognized without further measurement. Nevertheless, there are some cases in which a nominally quantitative result will be demanded, for

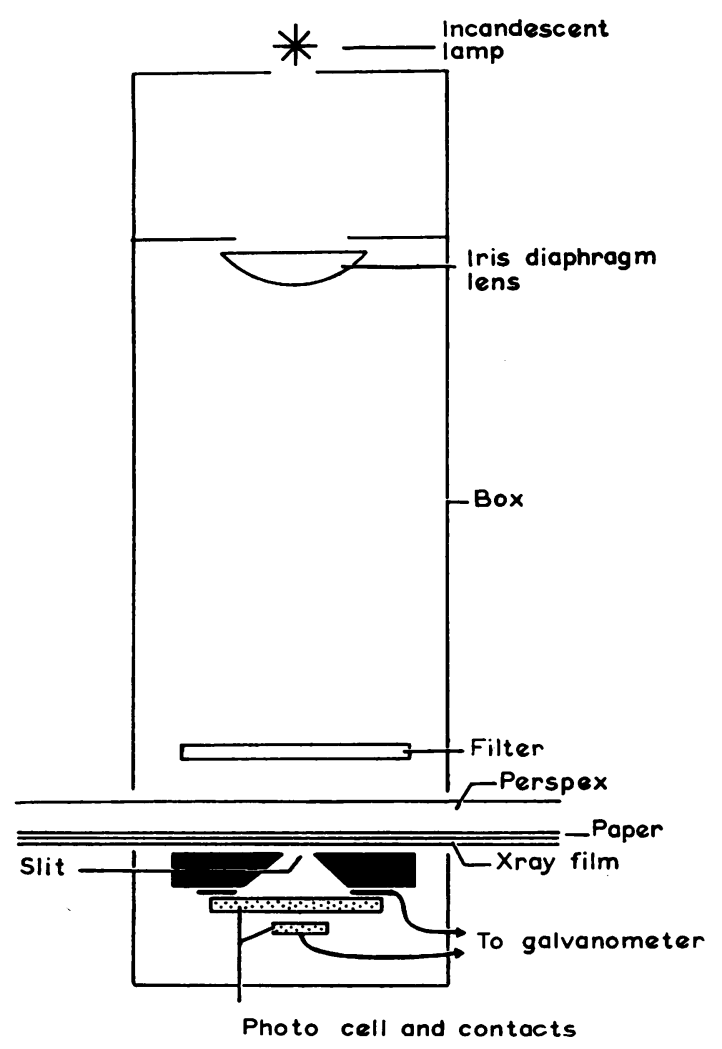

Fio. 1.--Scanning apparatus.

example, in the course of prolonged hepatitis. The apparatus we have used is indicated in Fig. 1 ; the image of an incandescent filament is formed on the paper strip just in front of a slit behind which is a photoelectric cell; the paper strip is traversed by hand across the slit, and the output from the photocell passed through a micrometer calibrated directly in extinction. The values of extinction are plotted against displacement along the paper.

The area under the curve is divided into component curves which represent the component protein species. It is very difficult to estimate these components, and many methods have been recommended. We have drawn in by eye curves which appear to be symmetrical and whose areas summate to the total area. The $\gamma$-globulin curve alone is not symmetrical, and its faster moving boundary is determined by the slower boundary of the $\beta$-globulin. The areas are measured with a planimeter, converted to percentages of the whole and to concentrations in grams per 100 $\mathrm{ml}$.

\section{Results}

The consistency of results was studied by performing five or more replicates on each of five sera. Table I shows the variability encountered;

TABLE I

RESULTS OF REPLICATES

\begin{tabular}{c|c|c|c|c|c}
\hline \multirow{2}{*}{$\begin{array}{c}\text { Sample } \\
\text { No. }\end{array}$} & \multirow{3}{*}{ Albumin } & \multicolumn{4}{|c}{ Globulins } \\
\cline { 2 - 6 } & & $a_{1}$ & \multicolumn{1}{|c}{$\alpha_{2}$} & \multicolumn{1}{c}{$\beta$} & $\gamma$ \\
\hline 1 & 20.39 & 8.44 & 7.74 & 7.59 & 55.84 \\
2 & 24.21 & 6.94 & 10.80 & 8.51 & 49.60 \\
3 & 23.88 & 7.12 & 8.61 & 12.45 & 47.94 \\
4 & 28.28 & 9.03 & 8.26 & 9.11 & 45.31 \\
5 & 25.49 & 8.17 & 9.55 & 9.94 & 46.86 \\
6 & 21.90 & 2.69 & 5.58 & 6.61 & 63.22 \\
7 & 24.92 & 2.83 & 6.50 & 4.23 & 61.53 \\
8 & 28.60 & 5.01 & 4.18 & 5.01 & 57.20 \\
9 & 29.71 & 2.94 & 5.02 & 6.39 & 55.95 \\
\hline Mean & 25.264 & 5.908 & 7.36 & 7.76 & 53.717 \\
\hline
\end{tabular}

Samples 1 to 5 inclusive were analysed on $0.02 \mathrm{ml}$., 6 to 9 on $0.01 \mathrm{ml}$. The systematic error is clearly shown. All samples were from a single blood specimen taken from a patient with cirrhosis of the liver.

when the total quantity of serum applied to the paper is reduced, then the apparent concentration of the minor components is reduced, while that of the major components increases. If a constant amount of serum is used, the coefficient of variation ranges from $8 \%$ for a component which constitutes about $50 \%$ of the serum protein to $20 \%$ for one which constitutes $10 \%$, and it is still greater for the lesser concentrations. These errors are very large, so large that it seems that changes in the minor components are not significant unless they are obvious to inspection of the strip by the eye ; certainly only the albumin and $\gamma$-globulin estimates are sufficiently reproducible to warrant quantification. 
Normal values are given in Table II ; each value is the mean of duplicates.

TABLE II

ELECTROPHORETIC ESTIMATES OF SERUM PROTEINS IN TEN APPARENTLY HEALTHY SUBJECTS

\begin{tabular}{|c|c|c|c|c|c|c|}
\hline \multirow{2}{*}{$\begin{array}{l}\text { Case } \\
\text { No. }\end{array}$} & \multirow{2}{*}{ Albumin } & \multicolumn{4}{|c|}{ Globulins } & \multirow{2}{*}{$\begin{array}{c}\text { Total } \\
\text { Protein }\end{array}$} \\
\hline & & $\alpha_{1}$ & $a_{2}$ & $\beta$ & $\gamma$ & \\
\hline$M\left\{\begin{array}{l}1 \\
2 \\
3 \\
4 \\
5 \\
6\end{array}\right.$ & $\begin{array}{l}3 \cdot 08 \\
2 \cdot 56 \\
2 \cdot 67 \\
3 \cdot 27 \\
3 \cdot 17 \\
2 \cdot 29\end{array}$ & $\begin{array}{l}0.40 \\
0.42 \\
0.50 \\
0.33 \\
0.32 \\
0.22\end{array}$ & $\begin{array}{l}0.67 \\
0.83 \\
0.66 \\
0.72 \\
0.62 \\
0.68\end{array}$ & $\begin{array}{l}1.09 \\
0.85 \\
1.21 \\
1.45 \\
1.18 \\
1.32\end{array}$ & $\begin{array}{l}1.78 \\
1.69 \\
2.36 \\
1.64 \\
1.72 \\
2.00\end{array}$ & $\begin{array}{l}7 \cdot 0 \\
6 \cdot 3 \\
7 \cdot 4 \\
7 \cdot 4 \\
7 \cdot 0 \\
6 \cdot 5\end{array}$ \\
\hline$F\left\{\begin{array}{r}7 \\
8 \\
9 \\
10\end{array}\right.$ & $\begin{array}{l}2.36 \\
2.67 \\
2.45 \\
2.53\end{array}$ & $\begin{array}{l}0.37 \\
0.29 \\
0.47 \\
0.45\end{array}$ & $\begin{array}{l}0.82 \\
0.67 \\
0.83 \\
1.07\end{array}$ & $\begin{array}{l}1.03 \\
1.13 \\
1.62 \\
1.32\end{array}$ & $\begin{array}{l}1.74 \\
1.94 \\
1.89 \\
2.14\end{array}$ & $\begin{array}{l}6 \cdot 3 \\
6 \cdot 7 \\
7 \cdot 25 \\
7 \cdot 5\end{array}$ \\
\hline Mean & $2 \cdot 705$ & 0.377 & 0.757 & $1 \cdot 22$ & 1.89 & \\
\hline $\begin{array}{c}\text { Standard } \\
\text { deviation }\end{array}$ & 0.3474 & 00877 & $0 \cdot 1347$ & $0 \cdot 2192$ & 0.22 & \\
\hline
\end{tabular}

Fractions as $\mathrm{g} . / 100 \mathrm{ml}$.

Among pathological sera the narrow based, tall peak, usually in the $\gamma$-globulin, sometimes in the $\beta$-globulin band, of myelomatosis, is diagnostic, and can be recognized very easily ; it often appears before the marrow aspirate is conclusive, and is sometimes present even when the total protein is normal or reduced. In nephrotic nephritis the $\alpha_{2}$-globulin is increased, while the albumin and $\gamma$-globulin are reduced; these changes may appear in the absence of symptoms and when the only sign is proteinuria. The commonest abnormality is a rise in $\gamma$-globulin, often accompanied by a fall in albumin; it occurs in chronic infection, infective hepatitis and hepatic cirrhosis, many reticuloses such as Hodgkin's disease, sarcoidosis, some leukaemias, rheumatoid arthritis, lupus erythematosus, and other collagen diseases. These changes are quite non-specific and cannot be related to any specific disease process or organsave that in renal disease there is usually a reduction, not an increase in $\gamma$-globulin. Since Kunkel's zinc sulphate turbidity test (1947) is claimed to provide a very reliable estimate of serum $\gamma$ globulin, we compared this test with our electrophoretic results.

A series of 45 sera was therefore investigated, duplicate estimates being made by both methods. The Kunkel reagent was prepared by dissolving $280 \mathrm{mg}$. barbitone and $210 \mathrm{mg}$. sodium barbitone in distilled water, adding $5 \mathrm{ml} .0 .48 \%$ zinc sulphate heptahydrate, and diluting to $1,000 \mathrm{ml}$. The reagent was kept in an automatic burette protected by a soda-lime tube, because previous experiments had shown that serious $p \mathbf{H}$ changes occurred if it were kept in a bottle and dispensed with a pipette. To $6 \mathrm{ml}$. reagent was added $0.1 \mathrm{ml}$. serum, using a capillary pipette and a teat to prevent possible change of $p \mathrm{H}$ if expired air were to enter the fluid, and after more than 10 but less than 120 minutes, the opacity was measured in a " unicam" D.G. spectrophotometer at $650 \mathrm{~m} \mu$. The standard, to which is ascribed the value of 20 units, was prepared by placing $3 \mathrm{ml}$. $1.15 \%$ barium chloride dihydrate in a dry $100 \mathrm{ml}$. flask and diluting to the mark with $0.25 \mathrm{~N}$ sulphuric acid ; it is important to adhere strictly to these conditions. A series of standards was prepared and the average accepted as the extinction corresponding to 20 units.

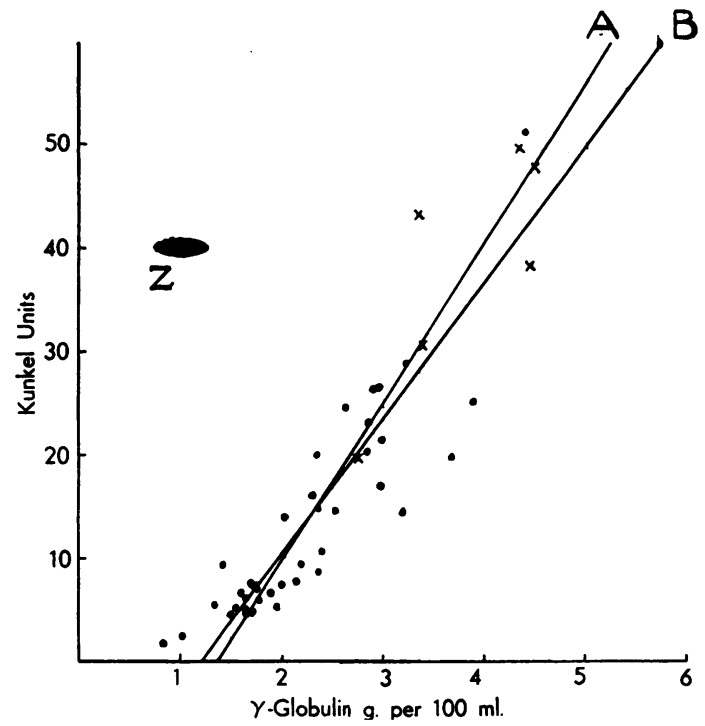

FIG. 2.-Scatter diagram of $\gamma$-globulin estimates and Kunkel's test. $A$, Regression line of $X$ (dependent) on $Y$ (independent). $B$, Regression line of $Y$ (dependent) on $X$ (independent). B, Ellipse of error showing twice the standard error along each $\mathbf{Z}$, Ellipse of error showing twice the standard error along each
axis. Points marked with $\mathbf{X}$ are from patients with myelomatosis.

A scatter diagram of the results is shown in Fig. 2, together with the regression lines. The lines are described by

$$
\begin{aligned}
& X=1.384+0.0647 Y(\text { Line A) } \\
& Y=12.913 X-15.159(\text { Line } B)
\end{aligned}
$$

where line $A$ tells us the best estimate of $X$ (the electrophoretic estimate of $\gamma$-globulin) given any value of $Y$ (Kunkel units of opacity) and line B tells us the best estimate of $Y$ given any value of $X$. The regression coefficient, $\tau$, is 0.914 , based on 45 pairs of observations, indicating that about $83 \%$ of the variance is due to the relation between 
$\mathrm{X}$ and $\mathrm{Y}$, and but $16 \%$ to other sources of variation.

The experimental error was deduced for $\mathrm{X}$ and $Y$ separately from the difference between each pair of observations, since a good estimate of the standard deviation between pairs is (for more than 20 pairs)

$$
\sigma=\frac{\overline{\mathrm{w}}}{1.13}
$$

where $\bar{w}$ is the mean of the differences; and the standard error of any mean based on two observations is given by

$$
\text { S.E. }=\frac{\tau}{\sqrt{2}}
$$

We find that for the Ys, $\sigma=0.5868$ and S.E. $=0.415$ units ; and for the Xs, $\sigma=0.1894$ and S.E. $=0.134$ units. As far as experimental error is concerned, the $95 \%$ confidence limits of any point on the scatter diagram can be illustrated by drawing an ellipse round each, using as semi-major axis $2 \times 0.134$ in the $X$ direction and as semiminor axis $2 \times 0.415$ in the $Y$ direction. Such an ellipse is drawn on the scatter diagram, and it is obvious that the total error is somewhat greater than that which can be ascribed to lack of reproducibility of the analytical techniques. There is no evidence on the relation between the true $\gamma$-globulin level and the estimates which are made of it by our techniques, and it is valueless to speculate on this matter.

Comparison of the zinc sulphate opacity measurements in our own laboratory with those made elsewhere on the same sample of serum suggests that agreement between laboratories, though usually close, is not exact, and that there can be quite gross differences. For this reason we suggest that the regression curve we quote applies only to our own reagents, which give, on the 10 normal sera of Table II, a mean of 6.62 units with a standard deviation of \pm 1.1653 ; the best estimate of the $95 \%$ confidence limits (using the $t$ test) is from 3.98 to 9.26 units, and for the $99 \%$ limits from 2.83 to 10.41 units.

\section{Summary}

The technique of filter-paper electrophoresis is laborious, and the precision of our version with aqueous staining not great.

It is useful particularly in the diagnosis of myelomatosis and of nephrotic nephritis.

The zinc sulphate turbidity test of Kunkel is simple and rapid; it correlates very closely with our estimation of $\gamma$-globulin, and is more suitable for studying the progress of a disease than is the more laborious electrophoresis.

We are indebted to Mr. F. Dewar for help in making our apparatus, and to Drs. J. Fielding, S. Shaw, and A. G. Signy for the gift of some sera.

\section{REFERENCES}

Crook, E. M., Harris, H., Hassan, F., and Warren, F. L. (1954). Biochem. J., 56, 434.

Kunkel, H. G. (1947). Proc. Soc. exp. Biol., N.Y., 66, 217.

Loeffer, W., and Wunderly, C. (1953). Journal of Clinical Pathology, 6, 282. 